\title{
Densities and partial molar volumes of dodecyltrimethylammonium bromide in binary systems (methanol+water) at $\mathrm{T}=(298.15$ to 323.15$) \mathrm{K}$
}

\author{
Sujit Kumar Shah, Ajaya Bhattarai", Sujeet Kumar Chatterjee \\ Department of Chemistry, M. M. A. M. C, Tribhuvan University, Biratnagar, Nepal
}

Email address:

bkajaya@yahoo.com (A. Bhattarai)

\section{To cite this article:}

Sujit Kumar Shah, Ajaya Bhattarai, Sujeet Kumar Chatterjee. Densities and Partial Molar Volumes of Dodecyltrimethylammonium Bromide in Binary Systems (Methanol+Water) at T=(298.15 to 323.15) K. American Journal of Chemical Engineering. Vol. 2, No. 6, 2014 , pp. 76-85. doi: 10.11648/j.ajche.20140206.12

\begin{abstract}
The densities of dodecyltrimethylammonium bromide in pure water and in methanol + water mixed solvent media containing $(0.10,0.20,0.30$, and 0.40$)$ volume fractions of methanol were measured at $(298.15,308.15,318.15$, and 323.15$) \mathrm{K}$. The concentrations are varied from $\left(0.4 \times 10^{-1}\right.$ to $\left.1.2 \times 10^{-1}\right) \mathrm{mol} \mathrm{kg}^{-1}$. The results showed almost increase on the densities with increasing surfactant concentration. Also, the densities are found to decrease with increasing temperature over the entire concentration range investigated in a given mixed solvent medium and these values are found to decrease with increasing methanol content in the solvent composition. The effects of concentration, solvent composition and temperature on the partial molar volumes are discussed.
\end{abstract}

Keywords: DTAB, Solvent Composition, Methanol-Water Mixed Solvent Media

\section{Introduction}

Various thermodynamic parameters have been studied in aqueous organic mixed solvent media and the effect of organic solvents have been discussed in literatures $(1,2,3,4)$. One of the research articles in aqueous organic mixed solvent media from our group on partial molar volumes of anionic surfactant sodium lauryl sulphate has been published (5). The experimental procedures have been used to calculate the partial molar volume for ionic and non-ionic organic compounds in aqueous solutions $(6,7)$. However, the evaluation of these procedures for surfactant is limited by the lack of availability of reliable experimental data of a broad variety of chemical structures and macromolecular characteristics. We have found the explanation of the partial molar volume in a number of papers in details $(8,9,10)$. Hence, only basic relation will be used on our system to calculate the partial molar volume. The partial molar volume, $V_{\mathrm{B}}$, is defined by the following equation;

$$
V_{\mathrm{B}}=(\partial V / \partial n)_{T, p}
$$

Where, $\partial V$ represent change in total volume and $\mathrm{n}$ as the number of moles. The partial molar volume is often provided in units of partial molar volume $\left(\mathrm{cm}^{3} / \mathrm{mol}\right)$. If there is concentration dependence, the partial molar volumes have to be extrapolated to concentration zero using one of the following two equations which calculate the apparent volume at the finite concentrations, $\mathrm{C}(10,11)$

$$
v=\frac{1}{\rho_{0}}-\frac{1}{c}\left(\frac{\rho}{\rho_{0}}-1\right)
$$

With $\mathrm{C}$ in $\mathrm{g} \mathrm{cm}^{-3}$ or

$$
V_{\mathrm{B}}=\frac{M}{\rho_{0}}-\frac{10^{3}}{c}\left(\frac{\rho}{\rho_{0}}-1\right)
$$

where, $\mathrm{M}$ is the molecular weight of the dodecyltrimethylammonium bromide, $\rho_{0}$ is the density of the solvent, $\rho$ is the density of the solution and $\mathrm{C}$ is equivalent concentration in $\mathrm{mol} \mathrm{kg}^{-1}$.

In order to calculate partial molar volumes, the solution densities are thoroughly measured for dodecyltrimethylammonium bromide at the temperatures $(298.15,308.15,318.15$, and 323.15$) \mathrm{K}$ in pure water and in methanol + water mixed solvent media containing $(0.10,0.20$, 
0.30 , and 0.40 ) volume fractions of methanol.

\section{Experimental Investigations}

\subsection{Chemicals and Materials}

Dodecyltrimethylammonium bromide was purchased from Loba Chemie Private Limited, Mumbai, India whereas methanol was purchased from Merck, India and was distilled with phosphorous pentoxide and then redistilled over calcium hydride. The purified solvent had a density of $0.77723 \mathrm{~g} \mathrm{~cm}^{-3}$ and a co-efficient of viscosity of $0.47424 \mathrm{mPa}$ s at $308.15 \mathrm{~K}$; these values are in good agreement with the literature values (12). Triply distilled water with a specific conductance less than $10^{-6} \mathrm{~S} \mathrm{~cm}^{-1}$ at $308.15 \mathrm{~K}$ was used for the preparation of the mixed solvents. The physical properties of methanol + water mixed solvents used in this study are shown in Table 1 and those values are matched with the published works $(5,13$, 14).

Table 1. Properties of methanol +water mixtures containing $(0.10,0.20,0.30$, and 0.40) volume fraction of methanol at $T=(298.15,308.15,318.15$, and 323.15) $K$

\begin{tabular}{rlll}
\hline \multicolumn{1}{c}{$\boldsymbol{T} / \mathbf{K}$} & $\rho_{0} /\left(\mathbf{g ~ c m}^{-\mathbf{3}}\right)$ & $\eta_{0} /(\mathbf{m P a} \mathbf{~})$ & $\boldsymbol{D}$ \\
\hline$\varphi_{1}=0.10$ & & & \\
298.15 & 0.98297 & 1.0844 & 75.09 \\
308.15 & 0.97973 & 0.8665 & 71.57 \\
318.15 & 0.97604 & 0.7017 & 68.18 \\
323.15 & 0.97438 & 0.6375 & 66.45 \\
$\varphi_{1}=0.20$ & & & \\
298.15 & 0.96963 & 1.3106 & 71.61 \\
308.15 & 0.96632 & 1.0217 & 68.14 \\
318.15 & 0.96162 & 0.8075 & 64.80 \\
323.15 & 0.95875 & 0.7300 & 63.15 \\
$\varphi_{1}=0.30$ & & & \\
298.15 & 0.95620 & 1.4712 & 67.65 \\
308.15 & 0.95160 & 1.1418 & 64.25 \\
318.15 & 0.94626 & 0.8957 & 60.99 \\
323.15 & 0.94331 & 0.8052 & 59.41 \\
$\varphi_{1}=0.40$ & & & \\
298.15 & 0.93957 & 1.4475 & 63.53 \\
308.15 & 0.93364 & 1.2034 & 57.18 \\
318.15 & 0.93140 & 0.9309 & 55.62 \\
323.15 & 0.92800 & 0.8288 &
\end{tabular}

\subsection{Density Measurements}

To calculate the partial molar volume of dodecyltrimethylammonium bromide in pure water, the density of pure water was used from the literatures $(15,16)$. The relative permittivity of methanol + water mixtures at the experimental temperatures were obtained by regressing the relative permittivity data as function of solvent composition from the literature (17).

The pycnometric method was used for measuring the density. The stock solutions were freshly prepared for each concentration series to avoid problems of aging and microorganism contamination, which was found to occur with diluted surfactant solutions (18).
The densities of solutions were determined by the use of Ostwald-Sprengel type pycnometer of about $25 \mathrm{~cm}^{3}$ capacity. The sample solution was transfused into the pycnometer by using a medical syringe. The pycnometer was then tightly fixed in a thermostat at the experimental temperatures within $\pm 0.005 \mathrm{~K}$. After thermal equilibrium was attained, the mass of the pycnometer was measured with electronic balance and the density was calculated. Density measurements are precise within $\pm 0.00005 \mathrm{~g} \mathrm{~cm}^{-3}$, which is satisfactory for our purpose. In order to avoid moisture pickup, all solutions were prepared in a dehumidified room with utmost care. In all cases, the experiments were performed in three replicates. The partial molar volumes at different molalities of the solutions are given in table 2 along with the standard error at $95 \%$ confidence interval.

\section{Results and Discussions}

Mixing methanol and water is exothermic as well as they occupy less volume than the sum of their volumes before mixing. The mixture was thoroughly shaken, and kept 24 hours for the released air bubbles to escape before attempting to make the solution of dodecyltrimethyl-ammonium bromide. Methanol + water is a popular mixed solvent and has been extensively studied (19). When methanol and water are mixed, the density is decreased with the increase of methanol content for the methanol + water mixed solvent system (Table 1).

The densities for the dodecyltrimethylammonium bromide in pure water and in four different methanol-water mixtures (containing 0.1, 0.2, 0.3, and 0.4 volume fractions of methanol) at $298.15,308.15,318.15$, and $323.15 \mathrm{~K}$ are depicted in Figures 1- 4 which show the variation of densities of the investigated solutions as a function of the surfactants concentration. From these Figures, it is evident that the densities exhibits almost increase with increasing concentration within the concentration range investigated here.

However, the density of the system increases with the addition of surfactant (Table 2). This behaviour has been found to be similar in the literatures $(5,20,21,22)$. It was also seen for density values for surfactants decrease with increasing temperature on Chauhan et al. work (22). Also, our density data for pure water of dodecyltrimethylammonium bromide match with R. De Lisi et al. Work (16).

Obviously, the concentration dependence of density follows the same pattern at all the temperatures and solvent composition investigated. In fact, the variations of density with DTAB concentrations are always found to be linear. We, therefore, determined the density of the solvent, by extrapolating the density values to zero DTAB concentration. It is very interesting to see our results of density of the solvent from Table 2 and calculated from the graph, Table 3 which is almost matching with each other. This shows that the density data of dodecyltrimethylammonium bromide in pure water and methanol -water mixed solvent media looks correct. 
The slopes of density versus DTAB concentration graph are always found to be positive in methanol-water mixtures, indicating strong ion-ion interactions in these media. Moreover, the slopes are found to increase in the mixed

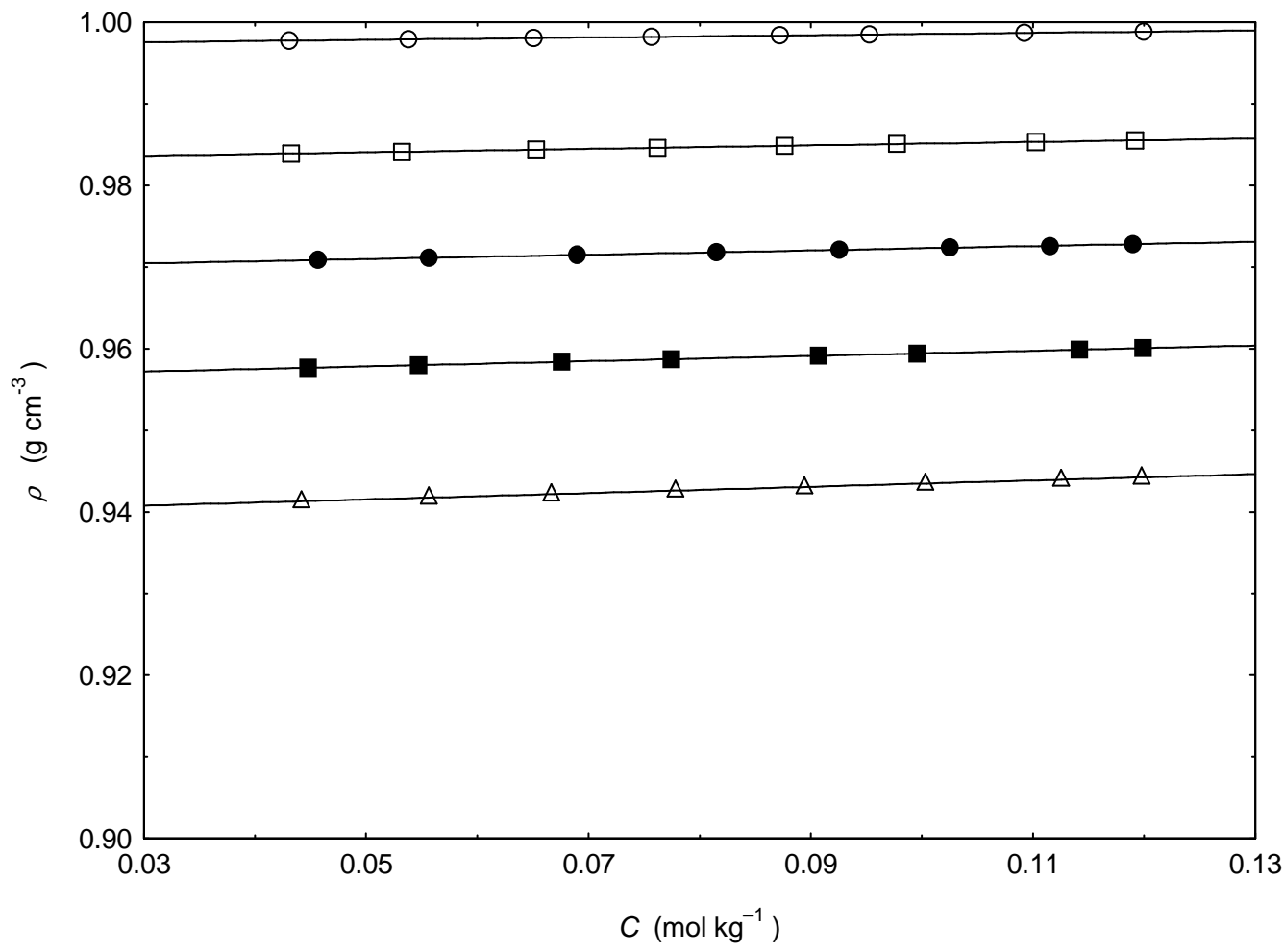

Figure 1. Concentration dependence of density for dodecyltrimethylammonium bromide at $298.15 \mathrm{~K}$, in pure water (open circles) and different methanol (1) + water (2) mixtures (open squares, 0.10 methanol; closed circles, 0.20 methanol; closed squares, 0.30 methanol; triangles, 0.40 methanol).

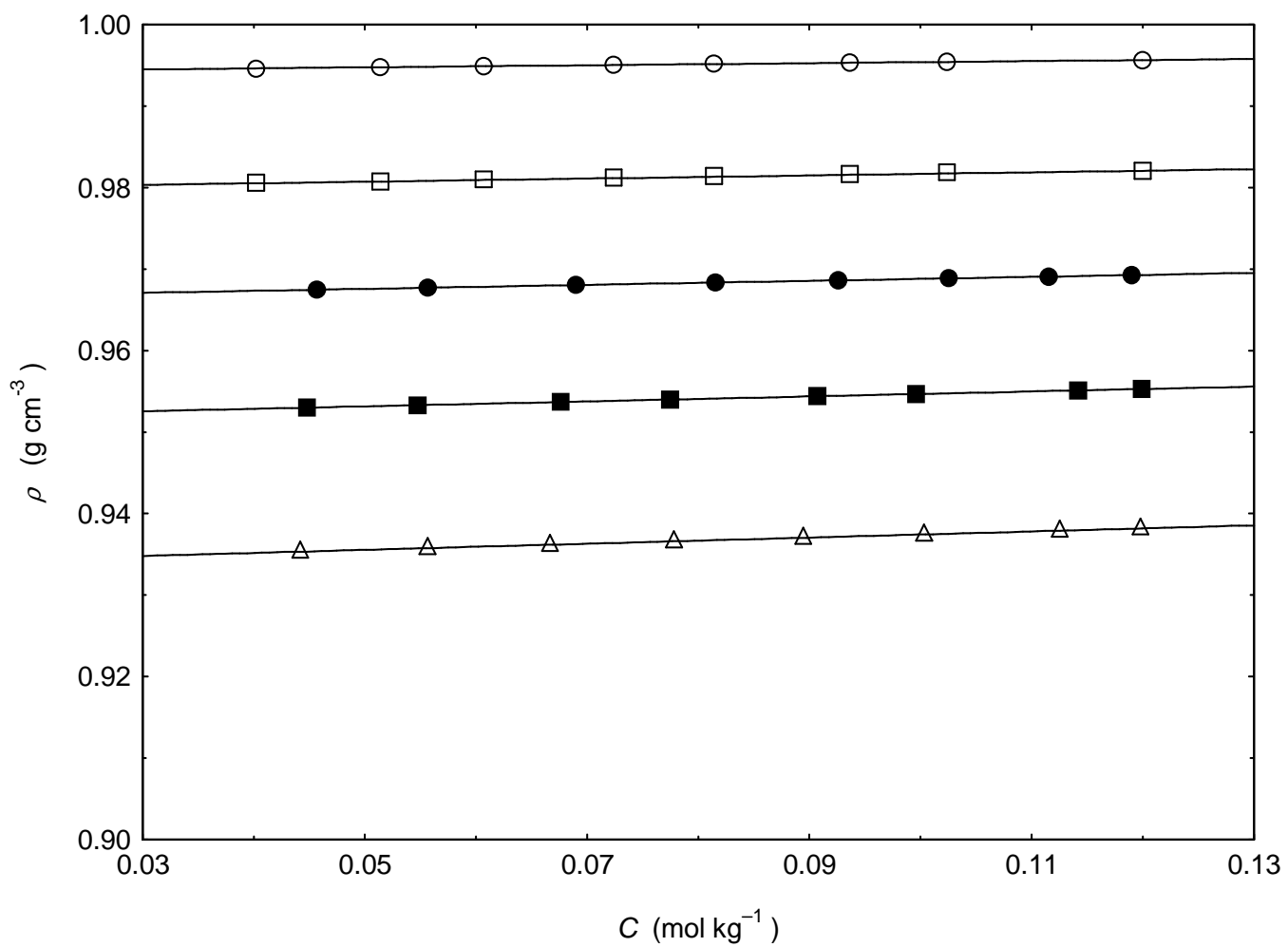

Figure 2. Concentration dependence of density for dodecyltrimethylammonium bromide at $308.15 \mathrm{~K}$, in pure water (open circles) and different methanol (1) + water (2) mixtures (open squares, 0.10 methanol; closed circles, 0.20 methanol; closed squares, 0.30 methanol; triangles, 0.40 methanol ). 


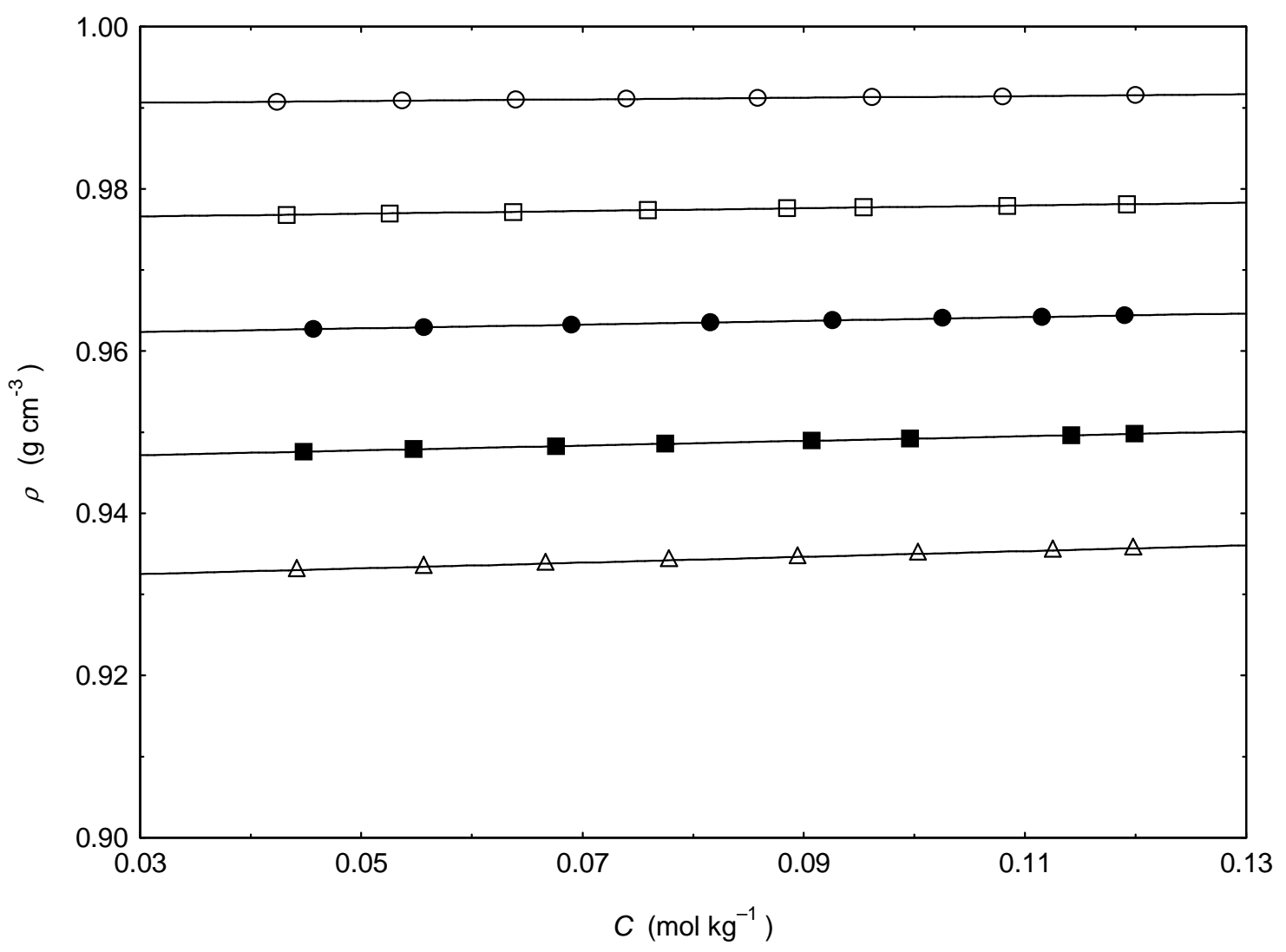

Figure 3. Concentration dependence of density for dodecyltrimethylammonium bromide at $318.15 \mathrm{~K}$, in pure water (open circles) and different methanol (1) + water (2) mixtures (open squares, 0.10 methanol; closed circles, 0.20 methanol; closed squares, 0.30 methanol; triangles, 0.40 methanol).

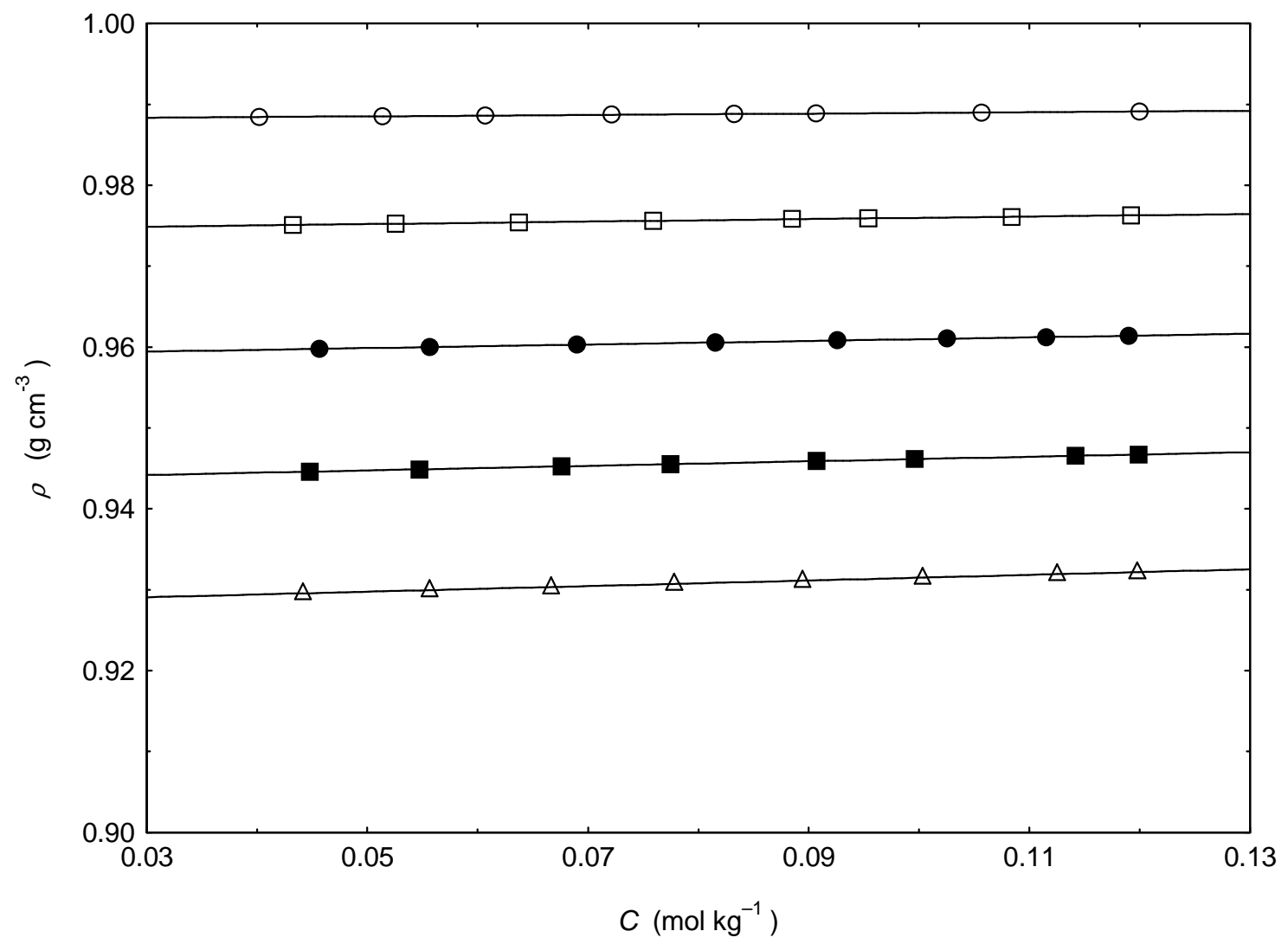

Figure 4. Concentration dependence of density for dodecyltrimethylammonium bromide at $298.15 \mathrm{~K}$, in pure water (open circles) and different methanol (1) + water (2) mixtures (open squares, 0.10 methanol; closed circles, 0.20 methanol; closed squares, 0.30 methanol; triangles, 0.40 methanol ). 
Table 2. Concentration, density and partial molar volume of dodecyltrimethylammonium bromide in pure water and methanol + water mixed solvent media at $T=(298.15,308.15,318.15$, and 323.15) $\mathrm{K}$

\begin{tabular}{|c|c|c|}
\hline$C /\left(\mathrm{mol} \mathrm{kg}^{-1}\right)$ & $\rho /\left(\mathrm{g} \mathrm{cm}^{-3}\right)$ & $V_{\mathrm{B}} /\left(\mathrm{cm}^{3} \mathrm{~mol}^{-1}\right)$ \\
\hline \multicolumn{3}{|l|}{$\mathrm{T}=298.15 \mathrm{~K}$} \\
\hline \multicolumn{3}{|l|}{$\phi_{1}=0.00$} \\
\hline 0.12000 & 0.99884 & $294.45 \pm 0.21$ \\
\hline 0.10926 & 0.99870 & $294.32 \pm 0.12$ \\
\hline 0.09530 & 0.99851 & $294.09 \pm 0.26$ \\
\hline 0.08725 & 0.99840 & $293.94 \pm 0.29$ \\
\hline 0.07569 & 0.99821 & $294.12 \pm 0.34$ \\
\hline 0.06508 & 0.99806 & $293.97 \pm 0.40$ \\
\hline 0.05384 & 0.99792 & $293.41 \pm 0.46$ \\
\hline 0.04311 & 0.99775 & $293.41 \pm 0.58$ \\
\hline \multicolumn{3}{|l|}{$\phi_{1}=0.10$} \\
\hline 0.11921 & 0.98552 & $291.91 \pm 0.22$ \\
\hline 0.11028 & 0.98535 & $291.73 \pm 0.23$ \\
\hline 0.09775 & 0.98509 & $291.62 \pm 0.25$ \\
\hline 0.08763 & 0.98486 & $291.74 \pm 0.29$ \\
\hline 0.07620 & 0.98462 & $291.67 \pm 0.34$ \\
\hline 0.06530 & 0.98441 & $291.26 \pm 0.35$ \\
\hline 0.05324 & 0.98412 & $291.71 \pm 0.40$ \\
\hline 0.04326 & 0.98392 & $291.37 \pm 0.60$ \\
\hline \multicolumn{3}{|l|}{$\phi_{1}=0.20$} \\
\hline 0.11900 & 0.97280 & $290.54 \pm 0.22$ \\
\hline 0.11154 & 0.97258 & $290.71 \pm 0.24$ \\
\hline 0.10256 & 0.97242 & $289.93 \pm 0.25$ \\
\hline 0.09260 & 0.97212 & $290.27 \pm 0.28$ \\
\hline 0.08156 & 0.97181 & $290.45 \pm 0.32$ \\
\hline 0.06898 & 0.97150 & $290.02 \pm 0.37$ \\
\hline 0.05566 & 0.97113 & $290.22 \pm 0.46$ \\
\hline 0.04569 & 0.97086 & $290.24 \pm 0.56$ \\
\hline \multicolumn{3}{|l|}{$\phi_{1}=0.30$} \\
\hline 0.11990 & 0.96006 & $288.78 \pm 0.22$ \\
\hline 0.11417 & 0.95988 & $288.76 \pm 0.23$ \\
\hline 0.09960 & 0.95942 & $288.66 \pm 0.26$ \\
\hline 0.09072 & 0.95916 & $288.34 \pm 0.29$ \\
\hline 0.07746 & 0.95872 & $288.44 \pm 0.34$ \\
\hline 0.06760 & 0.95840 & $288.42 \pm 0.40$ \\
\hline 0.05473 & 0.95798 & $288.45 \pm 0.47$ \\
\hline 0.04478 & 0.95766 & $288.38 \pm 0.58$ \\
\hline \multicolumn{3}{|l|}{$\phi_{1}=0.40$} \\
\hline 0.11980 & 0.94423 & $286.78 \pm 0.22$ \\
\hline 0.11253 & 0.94395 & $286.75 \pm 0.24$ \\
\hline 0.10032 & 0.94349 & $286.57 \pm 0.27$ \\
\hline 0.08945 & 0.94306 & $286.66 \pm 0.30$ \\
\hline 0.07782 & 0.94263 & $286.32 \pm 0.34$ \\
\hline 0.06666 & 0.94217 & $286.65 \pm 0.40$ \\
\hline 0.05562 & 0.94176 & $286.28 \pm 0.48$ \\
\hline 0.04416 & 0.94130 & $286.46 \pm 0.60$ \\
\hline \multicolumn{3}{|l|}{$\mathrm{T}=308.15 \mathrm{~K}$} \\
\hline \multicolumn{3}{|l|}{$\phi_{1}=0.00$} \\
\hline 0.12000 & 0.99563 & $297.19 \pm 0.21$ \\
\hline 0.10238 & 0.99540 & $297.21 \pm 0.25$ \\
\hline 0.09363 & 0.99533 & $296.73 \pm 0.27$ \\
\hline 0.08140 & 0.99521 & $296.21 \pm 0.31$ \\
\hline 0.07237 & 0.99506 & $296.57 \pm 0.35$ \\
\hline 0.06068 & 0.99491 & $296.41 \pm 0.42$ \\
\hline 0.05138 & 0.99478 & $296.45 \pm 0.50$ \\
\hline 0.04021 & 0.99460 & $297.13 \pm 0.65$ \\
\hline
\end{tabular}

\begin{tabular}{|c|c|c|}
\hline$C /\left(\mathrm{mol} \mathrm{kg}^{-1}\right)$ & $\rho /\left(\mathrm{g} \mathrm{cm}^{-3}\right)$ & $V_{\mathrm{B}} /\left(\mathrm{cm}^{3} \mathrm{~mol}^{-1}\right)$ \\
\hline \multicolumn{3}{|l|}{$\phi_{1}=0.10$} \\
\hline 0.11921 & 0.98204 & $294.97 \pm 0.29$ \\
\hline 0.11028 & 0.98189 & $294.73 \pm 0.23$ \\
\hline 0.09775 & 0.98166 & $294.58 \pm 0.27$ \\
\hline 0.08763 & 0.98144 & $294.79 \pm 0.30$ \\
\hline 0.07620 & 0.98123 & $294.65 \pm 0.35$ \\
\hline 0.06530 & 0.98104 & $294.26 \pm 0.39$ \\
\hline 0.05324 & 0.98077 & $294.76 \pm 0.48$ \\
\hline 0.04326 & 0.98059 & $294.43 \pm 0.58$ \\
\hline \multicolumn{3}{|l|}{$\phi_{1}=0.20$} \\
\hline 0.11900 & 0.96925 & $293.60 \pm 0.22$ \\
\hline 0.11154 & 0.96906 & $293.67 \pm 0.23$ \\
\hline 0.10256 & 0.96891 & $292.94 \pm 0.26$ \\
\hline 0.09260 & 0.96863 & $293.28 \pm 0.28$ \\
\hline 0.08156 & 0.96834 & $293.46 \pm 0.32$ \\
\hline 0.06898 & 0.96806 & $292.98 \pm 0.37$ \\
\hline 0.05566 & 0.96771 & $293.24 \pm 0.46$ \\
\hline 0.04569 & 0.96746 & $293.27 \pm 0.56$ \\
\hline \multicolumn{3}{|l|}{$\phi_{1}=0.30$} \\
\hline 0.11990 & 0.95528 & $291.77 \pm 0.22$ \\
\hline 0.11417 & 0.95510 & $291.80 \pm 0.23$ \\
\hline 0.09960 & 0.95467 & $291.65 \pm 0.27$ \\
\hline 0.09072 & 0.95442 & $291.36 \pm 0.35$ \\
\hline 0.07746 & 0.95400 & $291.46 \pm 0.34$ \\
\hline 0.06760 & 0.95370 & $291.39 \pm 0.39$ \\
\hline 0.05473 & 0.95330 & $291.41 \pm 0.50$ \\
\hline 0.04478 & 0.95299 & $291.33 \pm 0.90$ \\
\hline \multicolumn{3}{|l|}{$\phi_{1}=0.40$} \\
\hline 0.11980 & 0.93817 & $289.77 \pm 0.23$ \\
\hline 0.11253 & 0.93789 & $289.79 \pm 0.24$ \\
\hline 0.10032 & 0.93745 & $289.57 \pm 0.27$ \\
\hline 0.08945 & 0.93703 & $289.67 \pm 0.30$ \\
\hline 0.07782 & 0.93662 & $289.26 \pm 0.36$ \\
\hline 0.06666 & 0.93617 & $289.62 \pm 0.40$ \\
\hline 0.05562 & 0.93577 & $289.27 \pm 0.49$ \\
\hline 0.04416 & 0.93532 & $289.48 \pm 0.61$ \\
\hline \multicolumn{3}{|l|}{$\mathrm{T}=318.15 \mathrm{~K}$} \\
\hline \multicolumn{3}{|l|}{$\phi_{1}=0.00$} \\
\hline 0.12000 & 0.99154 & $300.45 \pm 0.22$ \\
\hline 0.10799 & 0.99140 & $300.53 \pm 0.23$ \\
\hline 0.09619 & 0.99132 & $300.04 \pm 0.27$ \\
\hline 0.08582 & 0.99120 & $299.99 \pm 0.30$ \\
\hline 0.07396 & 0.99110 & $299.62 \pm 0.35$ \\
\hline 0.06396 & 0.99098 & $299.70 \pm 0.40$ \\
\hline 0.05369 & 0.99087 & $299.52 \pm 0.47$ \\
\hline 0.04237 & 0.99073 & $299.67 \pm 0.62$ \\
\hline \multicolumn{3}{|l|}{$\phi_{1}=0.10$} \\
\hline 0.11921 & 0.97810 & $298.20 \pm 0.22$ \\
\hline 0.10839 & 0.97791 & $298.24 \pm 0.24$ \\
\hline 0.09539 & 0.97773 & $297.78 \pm 0.27$ \\
\hline 0.08850 & 0.97762 & $297.62 \pm 0.29$ \\
\hline 0.07590 & 0.97738 & $297.82 \pm 0.34$ \\
\hline 0.06373 & 0.97717 & $297.75 \pm 0.40$ \\
\hline 0.05256 & 0.97697 & $297.76 \pm 0.49$ \\
\hline 0.04326 & 0.97681 & $297.71 \pm 0.61$ \\
\hline \multicolumn{3}{|l|}{$\phi_{1}=0.20$} \\
\hline 0.11900 & 0.96437 & $296.60 \pm 0.22$ \\
\hline 0.11154 & 0.96419 & $296.69 \pm 0.23$ \\
\hline 0.10256 & 0.96406 & $295.91 \pm 0.25$ \\
\hline 0.09260 & 0.96379 & $296.28 \pm 0.28$ \\
\hline
\end{tabular}




\begin{tabular}{|c|c|c|}
\hline$C /\left(\mathrm{mol} \mathrm{kg}^{-1}\right)$ & $\rho /\left(\mathrm{g} \mathrm{cm}^{-3}\right)$ & $V_{\mathrm{B}} /\left(\mathrm{cm}^{3} \mathrm{~mol}^{-1}\right)$ \\
\hline 0.08156 & 0.96352 & $296.44 \pm 0.33$ \\
\hline 0.06898 & 0.96326 & $295.94 \pm 0.38$ \\
\hline 0.05566 & 0.96293 & $296.19 \pm 0.47$ \\
\hline 0.04569 & 0.96269 & $296.28 \pm 0.57$ \\
\hline \multicolumn{3}{|l|}{$\phi_{1}=0.30$} \\
\hline 0.11990 & 0.94979 & $294.75 \pm 0.23$ \\
\hline 0.11417 & 0.94962 & $294.76 \pm 0.23$ \\
\hline 0.09960 & 0.94920 & $294.67 \pm 0.27$ \\
\hline 0.09072 & 0.94896 & $294.39 \pm 0.30$ \\
\hline 0.07746 & 0.94856 & $294.46 \pm 0.34$ \\
\hline 0.06760 & 0.94827 & $294.42 \pm 0.39$ \\
\hline 0.05473 & 0.94789 & $294.41 \pm 0.50$ \\
\hline 0.04478 & 0.94759 & $294.45 \pm 0.59$ \\
\hline \multicolumn{3}{|l|}{$\phi_{1}=0.40$} \\
\hline 0.11980 & 0.93567 & $292.79 \pm 0.22$ \\
\hline 0.11253 & 0.93542 & $292.70 \pm 0.24$ \\
\hline 0.10032 & 0.93500 & $292.53 \pm 0.27$ \\
\hline 0.08945 & 0.93460 & $292.69 \pm 0.16$ \\
\hline 0.07782 & 0.93421 & $292.29 \pm 0.35$ \\
\hline 0.06666 & 0.93379 & $292.58 \pm 0.42$ \\
\hline 0.05562 & 0.93341 & $292.27 \pm 0.49$ \\
\hline 0.04416 & 0.93299 & $292.41 \pm 0.61$ \\
\hline \multicolumn{3}{|l|}{$\mathrm{T}=323.15 \mathrm{~K}$} \\
\hline \multicolumn{3}{|l|}{$\phi_{1}=0.00$} \\
\hline 0.12000 & 0.98910 & $302.97 \pm 0.21$ \\
\hline 0.10567 & 0.98898 & $302.88 \pm 0.24$ \\
\hline 0.09069 & 0.98890 & $302.25 \pm 0.28$ \\
\hline 0.08325 & 0.98883 & $302.22 \pm 0.31$ \\
\hline 0.07216 & 0.98873 & $302.12 \pm 0.35$ \\
\hline 0.06068 & 0.98862 & $302.09 \pm 0.42$ \\
\hline 0.05138 & 0.98853 & $302.03 \pm 0.49$ \\
\hline 0.04021 & 0.98841 & $302.25 \pm 0.63$ \\
\hline \multicolumn{3}{|l|}{$\phi_{1}=0.10$} \\
\hline 0.11921 & 0.97627 & $300.18 \pm 0.21$ \\
\hline 0.10839 & 0.97609 & $300.26 \pm 0.24$ \\
\hline 0.09539 & 0.97593 & $299.79 \pm 0.27$ \\
\hline 0.08850 & 0.97583 & $299.64 \pm 0.29$ \\
\hline 0.07590 & 0.97561 & $299.82 \pm 0.34$ \\
\hline 0.06373 & 0.97542 & $299.73 \pm 0.42$ \\
\hline 0.05256 & 0.97524 & $299.68 \pm 0.49$ \\
\hline 0.04326 & 0.97509 & $299.64 \pm 0.61$ \\
\hline \multicolumn{3}{|l|}{$\phi_{1}=0.20$} \\
\hline 0.11900 & 0.96138 & $298.57 \pm 0.23$ \\
\hline 0.11154 & 0.96120 & $298.69 \pm 0.23$ \\
\hline 0.10256 & 0.96108 & $297.91 \pm 0.25$ \\
\hline 0.09260 & 0.96082 & $298.29 \pm 0.28$ \\
\hline 0.08156 & 0.96056 & $298.47 \pm 0.32$ \\
\hline 0.06898 & 0.96031 & $298.01 \pm 0.37$ \\
\hline 0.05566 & 0.95999 & $298.33 \pm 0.49$ \\
\hline 0.04569 & 0.95977 & $298.23 \pm 0.85$ \\
\hline \multicolumn{3}{|l|}{$\phi_{1}=0.30$} \\
\hline 0.11990 & 0.94671 & $296.80 \pm 0.22$ \\
\hline 0.11417 & 0.94655 & $296.78 \pm 0.23$ \\
\hline 0.09960 & 0.94615 & $296.66 \pm 0.27$ \\
\hline 0.09072 & 0.94592 & $296.37 \pm 0.29$ \\
\hline 0.07746 & 0.94553 & $296.47 \pm 0.35$ \\
\hline 0.06760 & 0.94525 & $296.44 \pm 0.39$ \\
\hline 0.05473 & 0.94488 & $296.47 \pm 0.48$ \\
\hline 0.04478 & 0.94460 & $296.37 \pm 0.62$ \\
\hline \multicolumn{3}{|l|}{$\phi_{1}=0.40$} \\
\hline 0.11980 & 0.93217 & $294.77 \pm 0.23$ \\
\hline
\end{tabular}

\begin{tabular}{ccl}
\hline $\boldsymbol{C} /\left(\mathbf{m o l ~ k g}^{-\mathbf{1}}\right)$ & $\rho /\left(\mathrm{g} \mathrm{cm}^{-3}\right)$ & $\boldsymbol{V}_{\mathbf{B}} /\left(\mathbf{c m}^{\mathbf{3}} \mathbf{m o l}^{-\mathbf{1}}\right)$ \\
\hline 0.11253 & 0.93192 & $294.72 \pm 0.24$ \\
0.10032 & 0.93151 & $294.56 \pm 0.27$ \\
0.08945 & 0.93112 & $294.68 \pm 0.30$ \\
0.07782 & 0.93074 & $294.32 \pm 0.35$ \\
0.06666 & 0.93033 & $294.61 \pm 0.40$ \\
0.05562 & 0.92996 & $294.30 \pm 0.48$ \\
0.04416 & 0.92955 & $294.44 \pm 0.61$ \\
\hline
\end{tabular}

Table 3. Density of the solvent $\left(\rho_{0}\right)$, experimental slopes and the correlation coefficients of fits (as $r^{2}$ ) of dodecyltrimethylammonium bromide from Figures 1 to 4 in pure water and methanol-water mixtures at 298.15, $308.15,318.15$ and $323.15 \mathrm{~K}$.

\begin{tabular}{cccc}
\hline Vol. of methanol & slope & $\rho_{0}$ & $\mathbf{r}^{2}$ \\
\hline$T=298.15 \mathrm{~K}$ & & & \\
0 & 0.014 & 0.99714 & 0.9992 \\
0.1 & 0.021 & 0.98301 & 0.9996 \\
0.2 & 0.026 & 0.96966 & 0.9989 \\
0.3 & 0.032 & 0.95624 & 0.9998 \\
0.4 & 0.039 & 0.93960 & 0.9999 \\
$T=308.15 \mathrm{~K}$ & & & \\
0 & 0.013 & 0.99413 & 0.9933 \\
0.1 & 0.019 & 0.97976 & 0.9993 \\
0.2 & 0.024 & 0.96635 & 0.9987 \\
0.3 & 0.030 & 0.95164 & 0.9998 \\
0.4 & 0.037 & 0.93367 & 0.9998 \\
$T=318.15 \mathrm{~K}$ & & & \\
0 & 0.010 & 0.99032 & 0.9961 \\
0.1 & 0.017 & 0.97608 & 0.9986 \\
0.2 & 0.023 & 0.96166 & 0.9998 \\
0.3 & 0.029 & 0.94629 & 0.9998 \\
0.4 & 0.036 & 0.93143 & 0.9999 \\
$T=323.15$ & & & \\
0 & 0.009 & 0.98809 & 0.9911 \\
0.1 & 0.015 & 0.97443 & 0.9985 \\
0.2 & 0.022 & 0.95877 & 0.9983 \\
0.3 & 0.028 & 0.94335 & 0.9998 \\
0.4 & 0.035 & 0.92803 & 0.9999 \\
\hline
\end{tabular}

The effects of temperature and relative permittivity on the densities values are directly evident from Figures 1 - 4. At each temperature, the densities values are found to decrease with decreasing relative permittivity in going from 0.1 volume fractions of methanol to 0.4 volume fractions of methanol over the entire concentration range investigated. An increase in temperature, on the other hand, is found to decrease the density in a given solvent medium as manifested in these Figures. Evaluation of the solvent density lead to important insight as to the solution behavior of dodecyltrimethylammonium bromide. The solvent density values thus obtained along with the slopes and the correlation coefficients of fits, $\left(\right.$ as $^{2}$ ) are listed in Table 3.

Furthermore, at a given temperature, slopes are found to increase whereas the solvent density values are found to decrease as the solvent medium gets richer in methanol. Also, in all compositions; the solvent density, on the other hand, found to decrease with temperature (Table 3).

The partial molar volumes for the dodecyltrimethylammonium bromide in pure water and four 
other different methanol + water mixtures containing $(0.10$, $0.20,0.30$, and 0.40$)$ volume fraction of methanol at $(298.15$, $308.15,318.15$, and 323.15 ) $\mathrm{K}$ are shown in Table 2. Figures 5 to 8 show the variation of partial molar volumes of the investigated solution as a function of the surfactant concentration. From these Figures, the partial molar volumes exhibit almost independent with increasing concentration within the examined concentration ranges in this study.

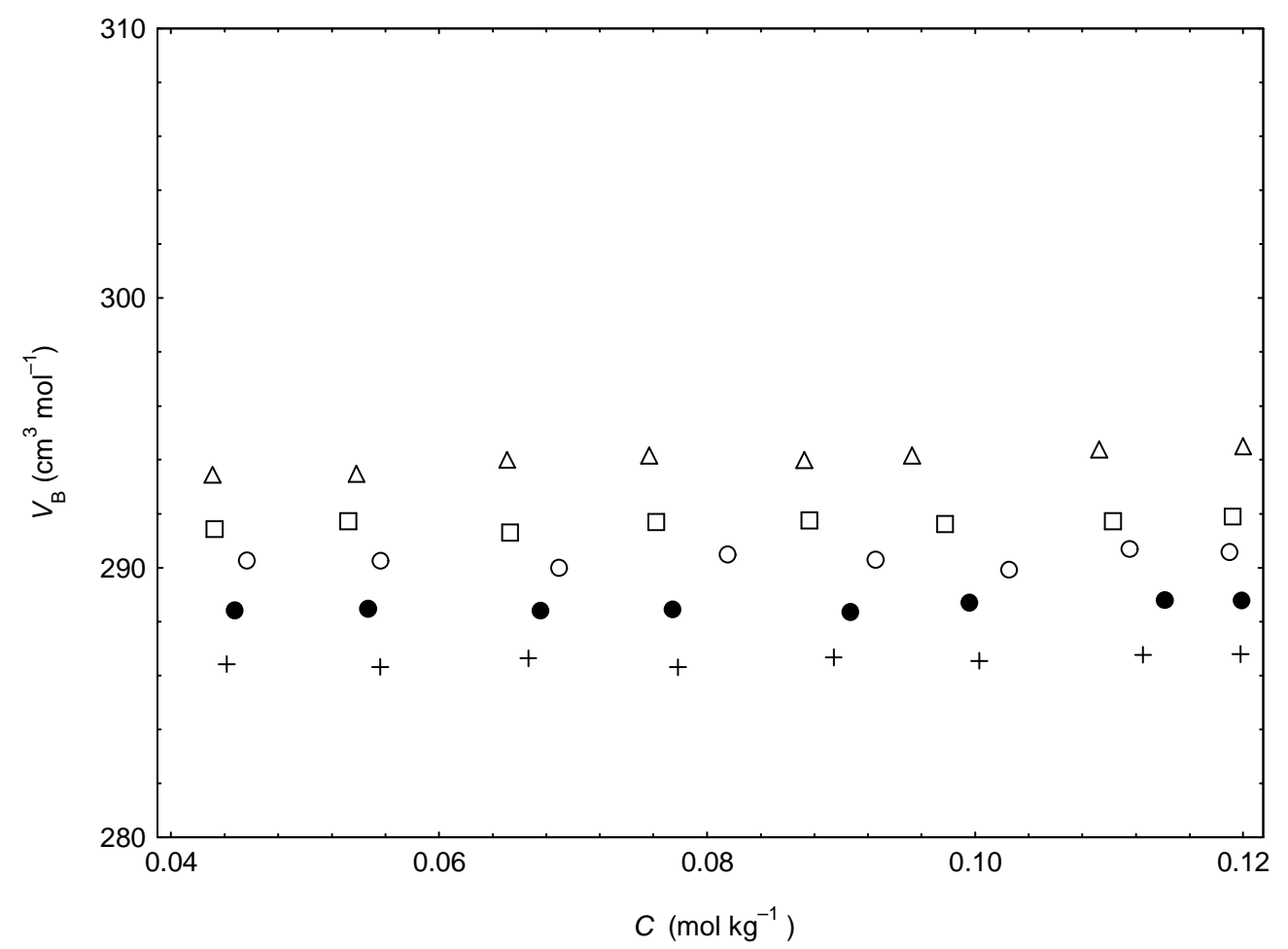

Figure 5. Concentration independence of partial molar volume for dodecyltrimethylammonium bromide at $298.15 \mathrm{~K}$, in pure water (triangles) and different methanol (1) + water (2) mixtures (squares, 0.10 methanol; circles, 0.20 methanol; closed circles, 0.30 methanol; crosses, 0.40 methanol).

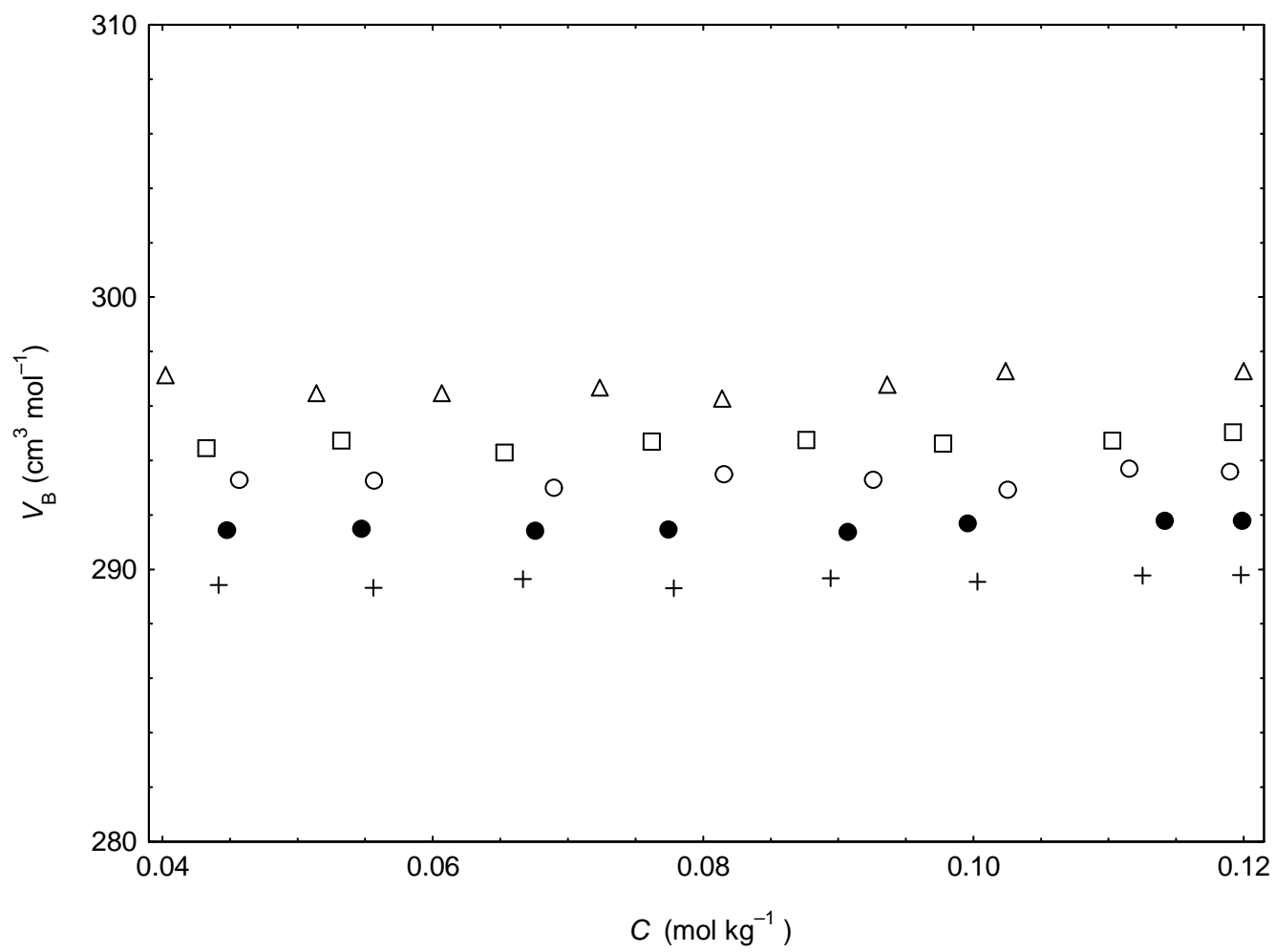

Figure 6. Concentration independence of partial molar volume for dodecyltrimethylammonium bromide at $308.15 \mathrm{~K}$, in pure water (triangles) and different methanol (1) + water (2) mixtures (squares, 0.10 methanol; circles, 0.20 methanol; closed circles, 0.30 methanol; crosses, 0.40 methanol ). 


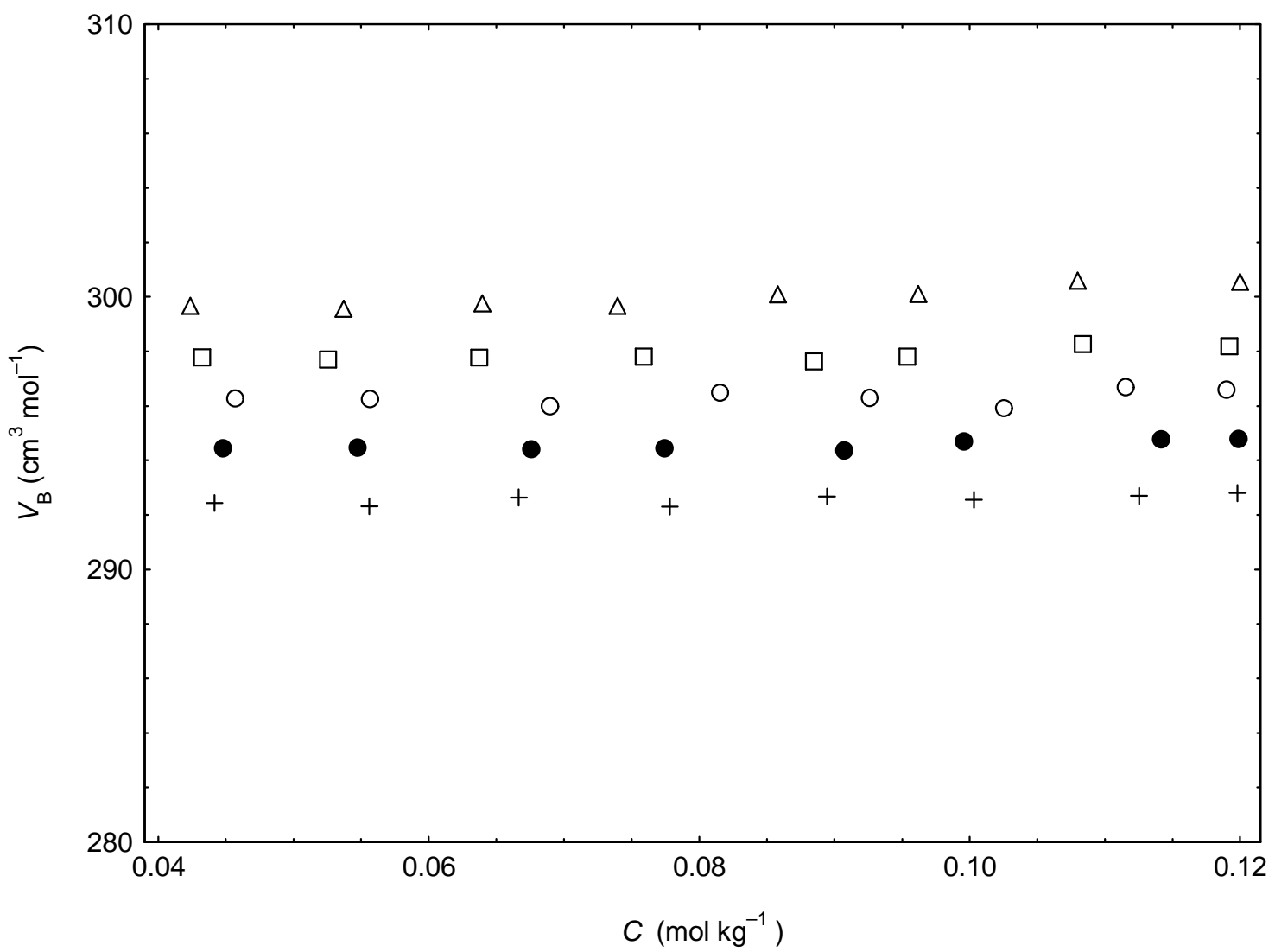

Figure 7. Concentration independence of partial molar volume for dodecyltrimethylammonium bromide at $318.15 \mathrm{~K}$, in pure water (triangles) and different methanol (1) + water (2) mixtures (squares, 0.10 methanol; circles, 0.20 methanol; closed circles, 0.30 methanol; crosses, 0.40 methanol).

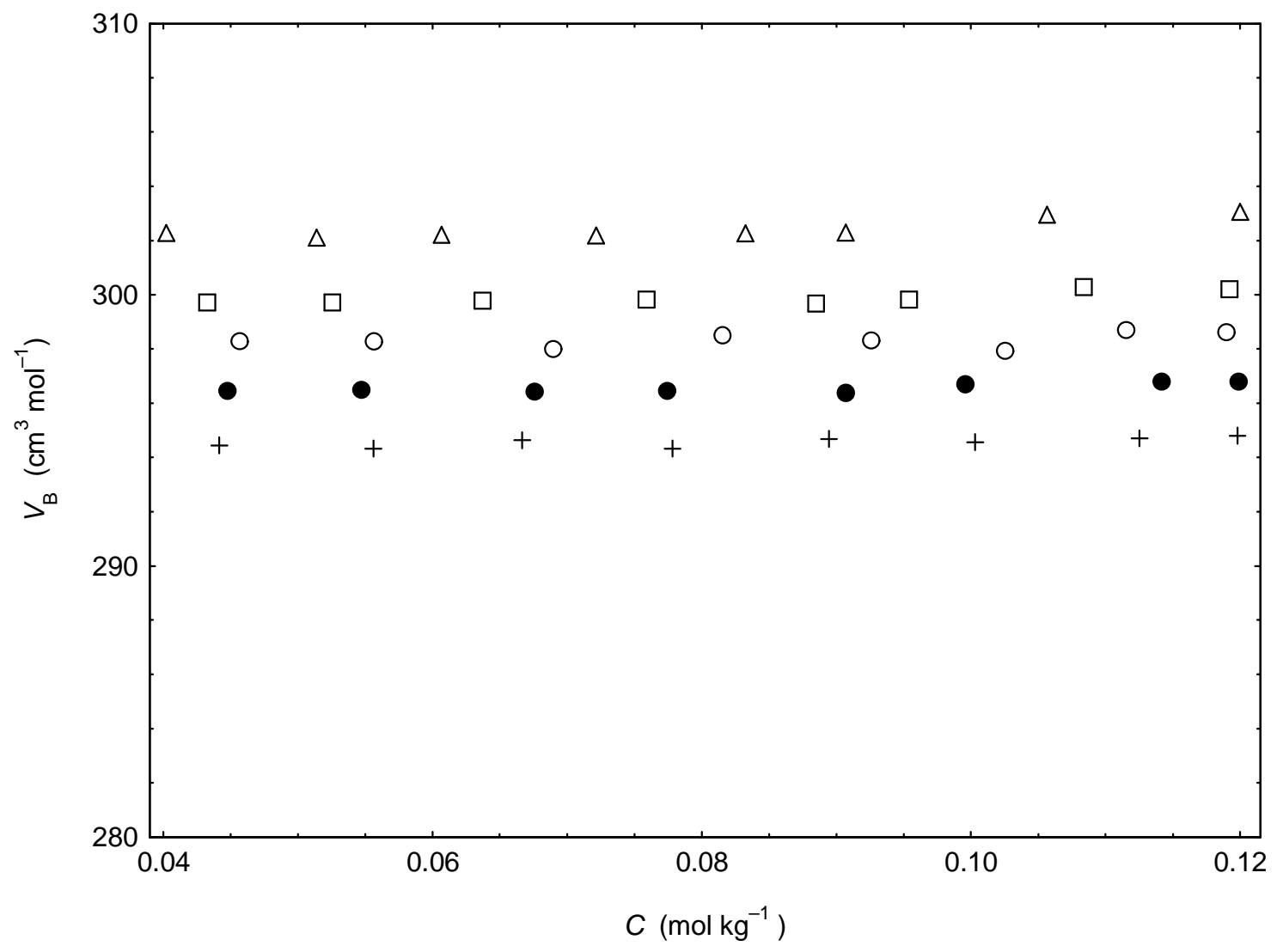

Figure 8. Concentration independence of partial molar volume for dodecyltrimethylammonium bromide at $323.15 \mathrm{~K}$, in pure water (triangles) and different methanol (1) + water (2) mixtures (squares, 0.10 methanol; circles, 0.20 methanol; closed circles, 0.30 methanol; crosses, 0.40 methanol ). 
Our partial molar volume data for pure water of dodecyltrimethylammonium bromide match with the previously published papers $(16,23)$. Obviously, the concentration independence of partial molar volumes follows the same pattern at all the temperatures and solvent compositions investigated.

The effects of temperature and relative permittivity on the partial molar volume values have been shown in Table 2. At each temperature, the partial molar volume values are found to decrease with decreasing relative permittivity by increasing the methanol content in the system. On the other hand, the partial molar volume is increased in the given system with increasing temperature. This is mostly due to the weakening of surfactant-solvent binding energy with increasing temperature. The same pattern has been also reported in the work (24).

The relative permittivity of the medium is decreased with increasing in the methanol content at a given temperature and similar findings were reported in the previous works also $(5$, $13,25,26)$.

\section{Conclusions}

Experimental results for the density of salt-free solution of a cationic surfactant dodecyltrimethylammonium bromide in pure water and methanol-water mixed solvent media have been presented as a function of surfactant concentration and temperature. The densities are found to decrease with increasing temperature over the entire concentration range investigated in a given mixed solvent medium whereas these values are also found to decrease as the relative permittivity of the medium decreases. Estimation of the slopes and the calculated solvent density provide important insight regarding the solution behavior of cationic surfactant in methanol-water mixtures. With the help of density measurement, the calculated partial molar volumes of dodecyltrimethylammonium bromide have been presented as a function of surfactant concentration and temperature. The partial molar volumes are found to increase with increasing temperature over the entire concentration range investigated in a given mixed solvent medium. Furthermore, at a particular temperature, the partial molar volumes are found almost same even at the different concentrations and these values are found to be decreased as the relative permittivity of the medium decreases.

\section{Acknowledgements}

This work was supported by University Grants Commission, Nepal. Sincere thanks to the Head of Department of Chemistry, Mahendra Morang Adarsh Multiple Campus, Biratnagar, Tribhuvan University, Nepal for providing the available research facilities to conduct this research work.

\section{References}

[1] M. L. Moya, A. Rodriguez, M. M. Graciani, G. Fernandez, Role of the solvophobic effect on micellization. Journal of Colloid and Interface Science, 2007. Vol. 316 (2), pp. 787 795.

[2] H. N. Singh, S. Swarup, S.M. Saleem, Effect of electrolytes on the micellization of ionic Surfactants in $n$-alkanol-water mixtures. Colloid and Interface Science, 1979. Vol. 68(1), pp. $128-134$.

[3] W. Li, Y. C. Han, J. L. Zhang, L. X. Wang, J. Song, Thermodynamic modeling of CTAB aggregation in waterethanol mixed solvents.Colloid Journal, 2006. Vol. 68. pp. $304-310$.

[4] K. Manna, A. K. Panda, Physicochemical Studies on the Interfacial and Micellization Behavior of CTAB in Aqueous Polyethylene Glycol Media. Journal of surfactants and detergents 2011. Vol. 14(4), pp. 563 - 576.

[5] A. Bhattarai, S.K. Chatterjee, T.K. Deo, T.P. Niraula, Effects of Concentration, Temperature, and Solvent Composition on the Partial Molar Volumes of Sodium Lauryl Sulfate in Methanol (1) + Water (2) Mixed Solvent Media. Journal of Chemical Engineering Data, 2011. Vol.56(8), pp. 3400 - 3405.

[6] H. Durchschlag, P. Zipper, Calculation of the partial volume of organic compounds and polymers Progress in Colloid Polymer Science. 1994. Vol. 94 (1), pp. 20-39.

[7] P. Gianni, L. Lepori, Group contributions to the partial molar volume of ionic organic solutes in aqueous solution. Journal of Solution Chemistry. 1996. Vol. 25(1), pp. 1 - 42.

[8] H. Eisenberg, In Biological Macromolecules and polyelectrolytes in Solution; Clarendron, Oxford 1976.

[9] H. Durchschlag, In Thermodynamic Data for Biochemistry and Biotechnology (ed. Hinz, H.-J.) Springer, Berlin. pp. 45, 1986.

[10] C. Wandrey, A. Bartkowiak, D. Hunkeler, Partial Molar and Specific Volumes of Polyelectrolytes: Comparison of Experimental and Predicted Values in Salt-free Solutions. Langmuir, 1999. Vol. 15(12), pp. 4062-4068.

[11] C. Tondre, R. Zana, Apparent molal volumes of polyelectrolytes in aqueous solution. Journal of Physical Chemistry, 1972. Vol. 76(23), pp. 3451 - 3459.

[12] H. Doe, T. Kitagawa, K, Sasabe, Conductometric study of some metal(II) perchlorates in methanol. Journal of Physical Chemistry, 1984. Vol. 88(15), pp. 3341 - 3345.

[13] A. Bhattarai, P. Nandi, B. Das, The Effects of Concentration, Relative Permittivity and Temperature on the Transport Properties of Sodium Polystyrenesulphonate in MethanolWater Mixed Solvent Media. Journal of Polymer Research, 2006, Vol. 13(1), pp. 475 - 482.

[14] A. Chatterjee, B. Das, Electrical Conductances of Tetrabutylammonium Bromide, Sodium Tetraphenylborate, and Sodium Bromide in Methanol (1) + Water (2) Mixtures at (298.15, 308.15, and 318.15) K. Journal of Chemical Engineeering Data, 2006, Vol. 51(4), pp. 1352 - 1355. 
[15] G. S. Kell, Precise representation of volume properties of water at one atmosphere. Journal of Chemical Engineering Data, 1967, Vol. 12(1), pp. 66 - 69.

[16] R. De Lisi, S. Milioto, R. E. Verrall, Volumes and compressibilities of pentanol in aqueous alkyltrimethylammonium bromide solutions at different temperatures. Journal of Solution Chemistry, 1990, Vol. 19(7), pp. 639 - 664.

[17] P. S. Albright, L. J. Gasting, Dielectric Constants of the Methanol-Water System from 5 to $55^{\circ}$. Journal of American Chemical Society, 1946, Vol. 68(6), pp. 1061-1063.

[18] A. Domard, M. Rinaudo, Preparation and characterization of fully deacetylated chitosan, International Journal of Biology Macromolecule, 1983, Vol. 5(1), pp. 49 - 52.

[19] F. Franks, J. Desnoyers, In Water Science Reviews 1; Franks, F., Ed.; Cambridge University Press: London,1985.

[20] S. K. Thakur, S. Chauhan, Ultrasonic Velocity and allied parameters of drug Colimax in aqueous 1-propanol at 298.15K Journal of Chemistry Pharmaceutical Research, 2011. Vol. 3(2), pp. 657 - 664.

[21] V. K. Syal, S. K. Thakur, S. Chauhan, P. Sharma, Ultrasonic Velocity Studies of Drug Parvon-spas in Mixed AlcoholWater Solvent Systems at $25^{\circ} \mathrm{C}$. International Journal of Thermophysics, 2005. Vol. 26(3), pp. 807 - 826.
[22] S. Chauhan, M.S. Chauhan, D. Kaushal, V.K. Syal, J. Jyoti, Study of Micellar Behavior of SDS and CTAB in Aqueous Media Containing Furosemide-A Cardiovascular Drug. Journal of Solution Chemistry, 2010. Vol. 39(5), pp. 622 - 638.

[23] M.F. Hossain, T.K. Biswas, M.N. Islam, M. E. Huque, Volumetric and viscometric studies on dodecyltrimethylammonium bromide in aqueous and in aqueous amino acid solutions in premicellar region. Monatshefte für Chemie, 2010. Vol. 141(12), pp. 1297-1308.

[24] M. J. Iqbal, M. Siddiquah, Partial Molar Volume of Mefenamic Acid in Alcohol at Temperatures between $\mathrm{T}=293.15$ and $\mathrm{T}=313.15 \mathrm{~K}$. Journal of Brazil Chemical Society, 2006. Vol. 17(5), pp. 851 - 858.

[25] A. Bhattarai, B. Das, Solvodynamic Properties of Sodium Polystyrenesulfo-nate in Methanol-Water Mixed Solvent Media in Absence and in the Presence of a Salt Using Viscometric Method, Macromolecular Symposia, 2012. Vol. 315(1), pp. 52 - 59.

[26] A. Chatterjee, B. Das, C. Das, Polyion-counterion interaction behavior for sodium carboxymethylcellulose in methanolwater mixed solvent media, Carbohydrate Polymer, 2012. Vol. 87(2), pp. 1144-1152. 